\title{
The Novel Neuroprotective Compound KMS99220 Has an Early Anti-neuroinflammatory Effect via AMPK and HO-1, Independent of Nrf2
}

\author{
Ji Ae Lee ${ }^{1}$, Hye Ri Kim¹, Jiyoung Kim², Ki Duk Park ${ }^{3}$, Dong Jin Kim ${ }^{4}$ and Onyou Hwang ${ }^{1 *}$ \\ ${ }^{1}$ Department of Biochemistry and Molecular Biology, University of Ulsan College of Medicine, Seoul 05505, ${ }^{2}$ Research Institute \\ for Veterinary Science, College of Veterinary Medicine, Seoul National University, Seoul 08826, ${ }^{3}$ Convergence Research Center \\ for Diagnosis, Treatment and Care System of Dementia, Korea Institute of Science and Technology, Seoul 02792, ${ }^{4}$ Center for \\ Neuro-Medicine, Brain Science Institute, Korea Institute of Science and Technology, Seoul 02792, Korea
}

\begin{abstract}
We have previously reported a novel synthetic compound KMS99220 that prevented degeneration of the nigral dopaminergic neurons and the associated motor deficits, suggesting a neuroprotective therapeutic utility for Parkinsons disease. Microglia are closely associated with neuroinflammation, which plays a key role in the pathogenesis of neurodegenerative diseases. In this study, we investigated the effects of KMS99220 on the signaling involving AMP-activated protein kinase (AMPK) and heme oxygenase-1 (HO-1), the enzymes thought to regulate inflammation. KMS99220 was shown to elevate the enzyme activity of purified AMPK, and phosphorylation of cellular AMPK in BV2 microglia. It increased the level of HO-1, and this was attenuated by AMPK inhibitors. KMS99220 lowered phosphorylation of IкB, nuclear translocation of NFKB, induction of inducible nitric oxide synthase, and generation of nitric oxide in BV2 cells that had been challenged with lipopolysaccharide. This anti-inflammatory response involved HO-1, because both its pharmacological inhibition and knockdown of its expression abolished the response. The AMPK inhibitors also reversed the anti-inflammatory effects of KMS99220. The induction of HO-1 by KMS99220 occurred within $1 \mathrm{~h}$, and this appeared not to involve the transcription factor Nrf2, because Nrf2 knockdown did not affect the compound's HO-1 inducing- and anti-inflammatory effects in this time window. These findings indicated that KMS99220 leads to AMPK-induced HO-1 expression in microglia, which in turn plays an important role in early anti-inflammatory signaling. Together with its neuroprotective property, KMS99220 may serve as a feasible therapeutic agent against neuroinflammation and neurodegeneration.
\end{abstract}

Key words: Microglia, Neuroinflammation, AMPK, HO-1, iNOS

\section{INTRODUCTION}

Neuroinflammation is mainly caused by microglia, the resident

Received August 16,2018, Revised October 10,2018,

Accepted October 11,2018

* To whom correspondence should be addressed.

TEL: 82-2-3010-4279, FAX: 82-2-3010-4248

e-mail: oyhwang@amc.seoul.kr immune cells of the central nervous system. Like macrophages, the major function of microglia is to remove cell debris and pathogens in response to injury or toxic insults. Activated, inflammatory microglia are neurotoxic, as they release various neurotoxic molecules such as nitric oxide (NO), TNF- $\alpha$ and IL- $1 \beta$, among others. If the activation status is continued due to dysfunction or aberrant activation, the consequent chronic neuroinflammation is thought to contribute to pathogenesis of neurodegenerative diseases such 
as Alzheimer's disease and Parkinson's disease (reviewed by [1]).

AMP-activated protein kinase (AMPK) is an enzyme involved in the regulation of cellular homeostasis and metabolic function. Accumulating evidence suggests that AMPK is also an important regulator of neuroinflammation. In microglial cells, direct pharmacological activation of AMPK lowered the lipopolysaccharide (LPS)-induced production of TNF- $\alpha$, IL- 6 and inducible NO synthase (iNOS) and nuclear translocation of NFKB [2,3]. In macrophages, overexpression of AMPK results in decreased inflammatory response, its knockdown leads to enhanced inflammatory response $[4,5]$, and activation of its signaling downregulates the function of NFKB system $[4,6]$. Hence, AMPK is considered as a potential therapeutic target in neuroinflammation-related diseases.

The phase-2 enzyme heme oxygenase-1 (HO-1) has also been shown to possess anti-inflammatory properties. Deficiency of HO- 1 exhibited abnormalities including chronic inflammation in mice [7], increased secretion of pro-inflammatory cytokines in activated mouse splenocytes [8], and hyperinflammation in human $[9,10]$. HO-1 induction in macrophages has been shown to mediate the switch from the proinflammatory M1 phenotype to the anti-inflammatory M2 phenotype [11]. In microglia, induction of HO-1 expression using phytochemicals or chemical agents has shown to mediate the resolution of inflammatory response [1215].

We recently synthesized a novel morpholine-containing chalcone compound KMS99220 (chemical structure shown in Fig. 7) that had a good pharmacokinetic profile and neuroprotective activity [16]. This compound exhibited excellent bioavailability and metabolic stability and no apparent side effect issues such as toxicity and cytochrome p450 inhibition. KMS99220 was shown to bind to Keapl protein, activate Nrf2, and induce expression of its target genes including HO-1 [16]. On the other hand, it has been reported that some chalcone compounds are anti-inflammatory [17-19] and can activate the AMPK pathway [20-23], and that AMPK can trigger HO-1 induction [24-26]. Taken together, we hypothesized that KMS99220, being a chalcone, might trigger AMPK activation and HO-1 expression in microglia resulting in modulation of neuroinflammatory responses.

\section{MATERIALS AND METHODS}

\section{Materials}

Fetal bovine serum, Dulbecco's modified Eagle’s medium, trypsin/EDTA, penicillin-streptomycin, and TRIzol reagent were from Thermo Fisher Scientific (Carlsbad, CA, USA). LPS, Compound $\mathrm{C}$ and adenine $9-\beta-\mathrm{D}$-arabinofuranoside (Ara $\mathrm{A})$ were purchased from Sigma-Aldrich (St. Louis, MO, USA). Control small interfering RNA (siRNA), HO-1 siRNA, Nrf2 siRNA and Lipofectamine RNAiMax reagent were purchased from Thermo Fisher Scientific. Tin protoporphyrin-IX (SnPP) was from Santa Cruz Biotechnology (Santa Cruz, CA, USA). Primary antibodies used are as follows: iNOS (sc-650), lamin B (sc-6216) and HO-1 (sc-10789) from Santa Cruz Biotechnology; NFkB (NBP1-96139) from Novus Biologicals (Littleton, CO, USA); IкB (\#9242), p-IкB (\#2859), AMPK (\#2532) and p-AMPK (\#2535) from Cell Signaling Technology (Danvers, MA, USA); and $\beta$-actin (A5441) from SigmaAldrich. Anti-rabbit IgG, anti-goat IgG and anti-mouse IgG were purchased from Sigma-Aldrich. First Strand cDNA Synthesis kit for RT-PCR was from MBI Fermentas (Ontario, Canada). Protein concentration of samples was determined by Bradford assay (Biorad, Hempstead, UK). Enhanced luminal-based chemiluminescence western blotting detection system was obtained from Pierce Chemical (Rockford, IL, USA). CellTiter-Glo ${ }^{\circledR}$ Luminescent Cell Viability Assay kit was from Promega (Madison, WI, USA).

\section{Synthesis of KMS99220}

KMS99220 was organically synthesized according to the method we had previously published [16].

\section{Cell cultures}

BV2 mouse microglial cells [27] were grown in Dulbeccos modified Eagle's medium with 10\% fetal bovine serum in the presence of $100 \mathrm{IU} / \mathrm{L}$ penicillin and $10 \mu \mathrm{g} / \mathrm{ml}$ streptomycin. Nrf2sh cells and GFPsh cells were produced and grown in culture media as previously reported by us [28]. The cells were maintained at $37^{\circ} \mathrm{C}$ in $95 \%$ air and $5 \% \mathrm{CO}_{2}$ in humidified atmosphere.

\section{Western blot analysis}

The cell lysates and nuclear fractions were obtained according to the method previously described [29]. Protein concentrations were determined, and equal amounts $(30 \mu \mathrm{g})$ of protein were separated on $10 \%$ SDS polyacrylamide gel and transferred onto polyvinylidene difluoride-nitrocellulose membrane. The membranes were blocked for $1 \mathrm{~h}$ in $20 \mathrm{mM}$ Tris- $\mathrm{HCl}$ containing $137 \mathrm{mM}$ $\mathrm{NaCl}, 0.05 \%$ Tween 20 and 5\% skim milk at room temperature, incubated overnight with primary antibody against iNOS (1:200),

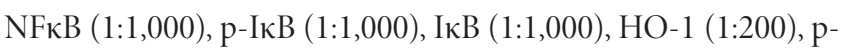
AMPK (1:1,000), AMPK (1:1,000), lamin B (1:200) or $\beta$-actin $(1: 60,000)$ at $4^{\circ} \mathrm{C}$ followed by horseradish peroxidase-conjugated secondary antibodies for $1 \mathrm{~h}$ at room temperature. Protein bands were visualized using chemiluminescence substrate and quantitatively analyzed by densitometry. 


\section{AMPK kinase assay}

The activity of AMPK enzyme was determined in the presence or absence of KMS99220 using purified AMPK protein (service provided by Eurofins Scientific, Dundee, UK). The purified AMPK was first incubated with KMS99220 in an assay buffer containing $8 \mathrm{mM}$ MOPS (pH 7.0), 0.2 mM EDTA, $200 \mu \mathrm{M}$ AMP, and $100 \mu \mathrm{M}$ of the substrate AMARAASAAALARRR. The reaction was initiated by the addition of $\mathrm{Mg} / \mathrm{ATP} \operatorname{mix}$ (final concentrations, $10 \mathrm{mM}$ magnesium acetate and $45 \mu \mathrm{M}\left[\gamma^{-3}{ }^{33} \mathrm{P}\right]$-ATP). After incubation for $40 \mathrm{~min}$ at room temperature, the reaction was stopped by the addition of phosphoric acid (final concentration, $0.5 \%$ ). Ten $\mu$ of the reaction was then spotted onto a P30 filtermat and washed four times for $4 \mathrm{~min}$ in $0.425 \%$ phosphoric acid and once in methanol prior to drying and scintillation counting.

\section{Cytotoxicity assays}

Cell viability was assessed by determining the intracellular level of ATP using CellTiter-Glo ${ }^{\circledR}$ kit as described before [29]. total RNA and the First Strand cDNA Synthesis kit following the manufacturer's directions. PCR was performed at $94^{\circ} \mathrm{C}$ for $30 \mathrm{sec}$, $55^{\circ} \mathrm{C}$ for $40 \mathrm{sec}$, and $72^{\circ} \mathrm{C}$ for $1 \mathrm{~min}$ for 25 cycles using specific primers: iNOS (forward, 5'-ATGTCCGAAGCAAACATCAC-3'; reverse, 5'TAATGTCCAGGAAGTAGGTG-3'), HO-1 (forward, 5'-AGCAGGACATGGCCTCT-3'; reverse, 5'-TCTGTCAGCATCACCTGCAG-3'), NAD(P)H:quinone oxidoreductase 1 (NQO1) (forward, 5'-CCATCCTAAACAGCGATCA-3'; reverse, 5'-TAGCTTTGATCTGGTTGTC-3'), glutamate-cysteine ligase modifier subunit (GCLM) (forward, 5'AGCTGGACTCTGTGATCATGGCTT-3'); reverse, 5'-CAAAGGCAGTCAAATCTGGTGGCA-3'), glutamate-cysteine ligase catalytic subunit (GCLC) (forward, 5'-ATGACTGTTGCCAGTGGATGAGA-3'; reverse, 5'-ACACGCCATCCTAAACAGCGATCA-3'), Nrf2 (forward, 5'-CTCGCTGGAAAAAGAAGTGG-3'; reverse, 5'-CCGTCCAGGAGTTCAGAGAG-3'), and glyceraldehydes-3-phosphate dehydrogenase (GAPDH) (forward, 5'-CACCACCATGGAGAAGGCTGG-3'; reverse,5'-TTGTCATGGATGACCTTGGCCAGG-3').

\section{RT-PCR}

Reverse transcription reactions were performed using $5 \mu \mathrm{g}$ of

A

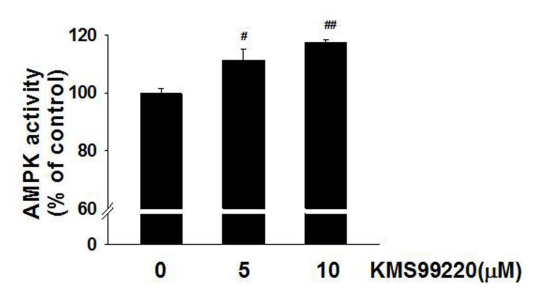

C

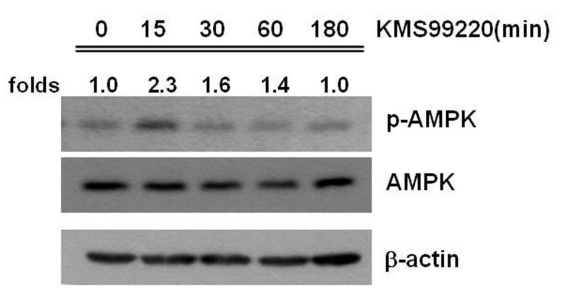

E

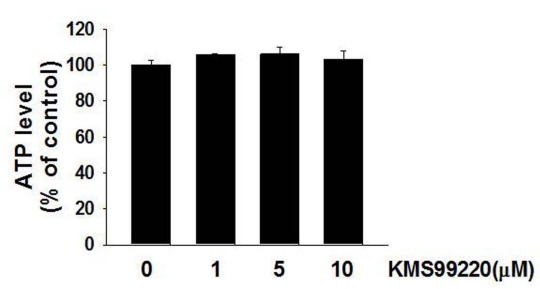

B

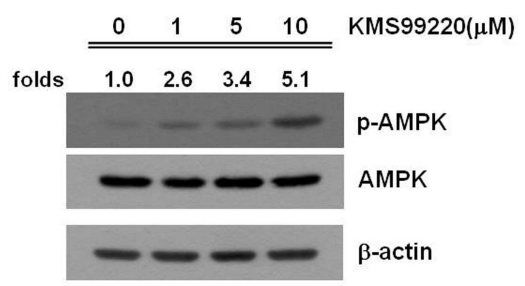

D

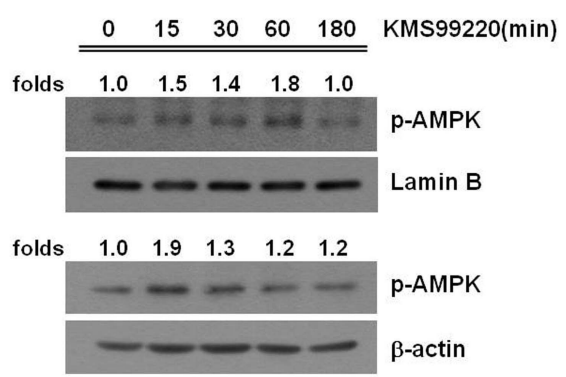

Fig. 1. KMS99220 activates the AMPK signaling pathway in microglial cells. (A) AMPK activity in the presence of KMS99220 was measured using a radiometric assay as described in Methods. The data are expressed as $\%$ of untreated control \pm SEM; ${ }^{*} \mathrm{p}<0.05$ and ${ }^{* *} \mathrm{p}<0.01$. (B) BV2 cells were treated with 1,5 and 10 $\mu \mathrm{M}$ KMS99220 for 15 min. (C, D) BV2 cells were treated with $10 \mu \mathrm{M}$ KMS99220 for 15, 30, 60 and $180 \mathrm{~min}$. Western blot analysis was performed against $\mathrm{p}$ AMPK, AMPK, $\beta$-actin and Lamin B. For quantitation, values obtained from densitometry were normalized against respective loading controls (AMPK for B and $C$; $\beta$-actin or Lamin B for D) and are expressed as induction fold of untreated control, as indicated above each panel. (D) Nuclear and cytoplasmic extracts were subjected to western blot analysis against p-AMPK. Lamin B was used as a loading control and nuclear marker. (E) BV-2 cells were exposed to various concentrations of KMS99220 for $24 \mathrm{~h}$, and cytoxicity was assessed by ATP assay. 


\section{Griess assay}

Cells were treated with various concentrations of KMS99220 with $0.2 \mu \mathrm{g} / \mathrm{ml}$ LPS. After $24 \mathrm{~h}, 100 \mu \mathrm{l}$ of culture medium was mixed with $50 \mu \mathrm{l}$ of Griess reagent ( $1 \%$ sulfanilamide, $0.1 \%$ naphthylethlyene diamine dihydrochloride and $2 \%$ phosphoric acid) and incubated at room temperature for $10 \mathrm{~min}$. The nitrite level was measured at $540 \mathrm{~nm}$ with a microplate reader (SPECTRA MAX 340 pc; Molecular Devices, Menlo Park, CA, USA).

\section{siRNA transfection}

Cells were transiently transfected with siRNA (final concentration of $40 \mathrm{nM}$ ) for HO-1, Nrf2 or control using Lipofectamine RNAiMax reagent according to the manufacturer's instructions. After $24 \mathrm{~h}$, the cells were treated with KMS99220 or LPS, and then RT-PCR, western blot analysis and Griess assay were conducted.

\section{Data analyses}

Statistical tests were carried out using PRISM (GraphPad Software, San Diego, CA, USA). A value of $\mathrm{p}<0.05$ was considered statistically significant. Comparisons of three or more groups were analyzed by one-way ANOVA (analysis of variance) and post Dunnett's multiple comparison tests.

\section{RESULTS}

\section{KMS99220 activates the AMPK signaling pathway in mi- croglia}

We first examined whether our chalcone compound KMS99220 might activate AMPK. When purified AMPK protein was exposed to KMS99220, the activity of the enzyme was increased in a concentration dependent manner, with $17 \%$ elevation at $10 \mu \mathrm{M}$ (Fig. 1A). When the murine microglial BV2 cells were exposed to KMS99220, an increase in the phosphorylated AMPK was observed in a manner dependent on KMS99220 concentration (Fig. 1B). This occurred within 15 min of the exposure, after which the level was restored to that of the untreated control (Fig. 1C). In accordance with the previous report that activated AMPK translocates into the nucleus [30], the KMS99220 treatment resulted in accumulation of the phospho-AMPK in the nucleus, between 15 and $60 \mathrm{~min}$ (Fig. 1D). KMS99220 had no cytotoxicity in the concentration range tested (Fig. 1E).

\section{AMPK is involved in microglial HO-1 induction by KMS99220}

We tested if KMS99220 might induce HO-1 expression in microglial cells, and if so, whether this might involve AMPK. We performed RT-PCR, because we had previously demonstrated that the results of RT-PCR corresponded well to those of real-time RT-

A

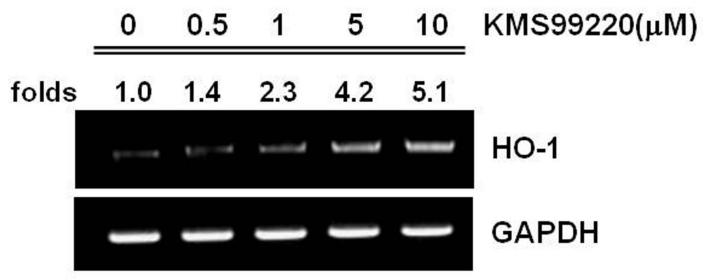

folds $\quad \begin{array}{lllll}1.0 & 1.5 & 1.6 & 2.7 & 4.3\end{array}$

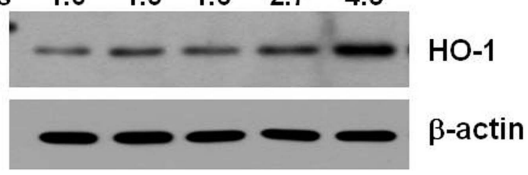

B

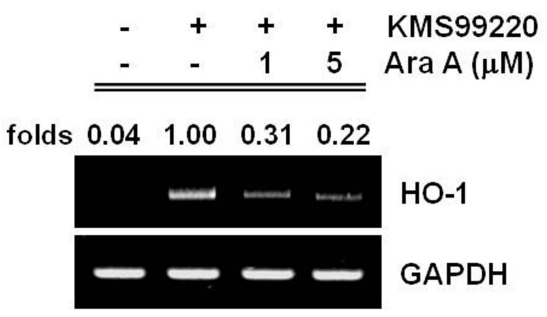

Fig. 2. AMPK is involved in HO-1 induction by KMS99220. (A) BV2 cells were treated with various concentrations of KMS99220 for $6 \mathrm{~h}$ or $24 \mathrm{~h}$, and RT-PCR and western blot analysis was performed against HO-1. For quantitation, values obtained from densitometry were normalized against respective loading controls (GAPDH or $\beta$-actin) and are expressed as induction fold of untreated control, as indicated above each panel. (B) BV2 cells were pretreated with Ara A or Compound C for $1 \mathrm{~h}$, KMS99220 was added to the final concentration of $10 \mu \mathrm{M}$, and the cells were further cultured for $6 \mathrm{~h}$. RT-PCR was performed against HO-1 using GAPDH as a loading control. For quantitation, values obtained from densitometry were normalized against GAPDH and are expressed as fold of KMS99220-treated control. 
PCR for all genes investigated in the present study under our experimental conditions [18]. As shown in Fig. 2A, KMS99220 dramatically and dose-dependently elevated the mRNA and protein levels of HO-1. On the other hand, when the cells were pretreated with the AMPK pharmacological inhibitors Ara A or Compound C, the KMS99220-induced HO-1 elevation was not as apparent (Fig. 2B). This suggested that AMPK played a role in the HO-1 induction.

\section{KMS99220 suppresses $\mathrm{NF}$ B signaling via HO-1 induction}

Since KMS99220 induced the HO-1 expression, it was possible that it might also suppress the signaling of the pro-inflammatory transcription factor NFkB. Western blot analysis showed that nuclear NFkB, which had been elevated upon exposure to the inflammagen LPS, was suppressed in the cells treated with KMS99220 (Fig. 3A). Since NFкB is known to be activated through phosphorylation and the subsequent degradation of its cytosolic inhibitor protein IкB [31], we asked if KMS99220 might also modulate this upstream step. As shown in Fig. 3B, KMS99220 indeed blocked the elevation of phospho- $\mathrm{I} \kappa \mathrm{B}$. The decrease in the total I $\mathrm{KB}$ protein level after the LPS exposure, implicating degradation of phosphoI $\mathrm{B}$, was also not as apparent in the cells treated with KMS99220, supporting the notion that phosphorylation of I $\kappa \mathrm{B}$ has been compromised by KMS99220.

We tested whether this effect of KMS99220 on the $\mathrm{I} \kappa \mathrm{B} / \mathrm{NF} \kappa \mathrm{B}$ system might be mediated by HO-1. For this, HO-1 expression was knocked down by transfection of BV2 cells with siRNA (Fig. 3C). As shown in Fig. 3D, in the cells whose HO-1 expression was obliterated, KMS99220 was no longer able to inhibit the LPSinduced I $\mathrm{KB}$ phosphorylation, and there was no significant difference between the LPS-alone and the LPS+KMS99220-treated cells. This was in contrast to the cells transfected with the control siRNA, in which LPS+KMS99220 treatment lowered the level of phosphorylated I $\kappa$ B. This phenomenon was also confirmed using a pharmacological approach. When BV2 cells were pretreated with the HO-1 inhibitor SnPP, the inhibitory effect of KMS99220 on $\mathrm{I} \kappa \mathrm{B}$ phosphorylation was reversed, and this occurred in a dosedependent manner (Fig. 3E).
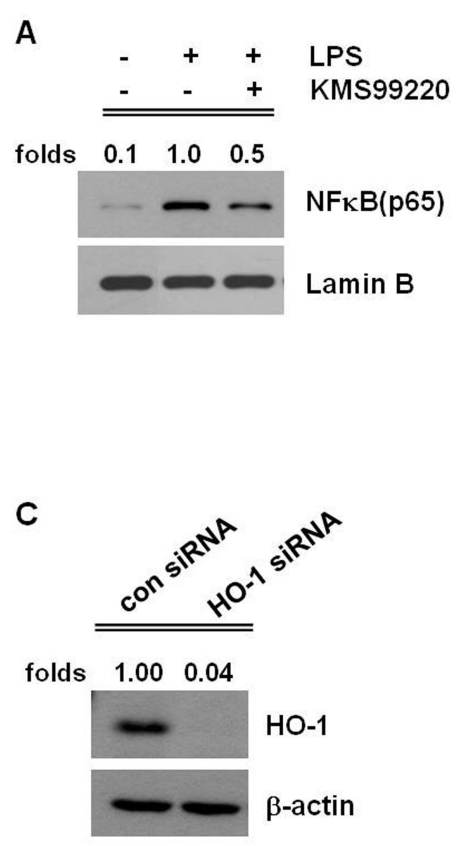

E

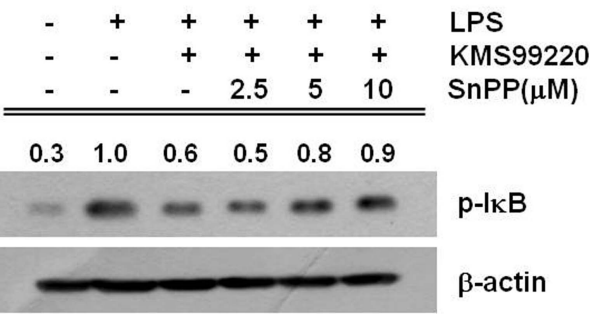

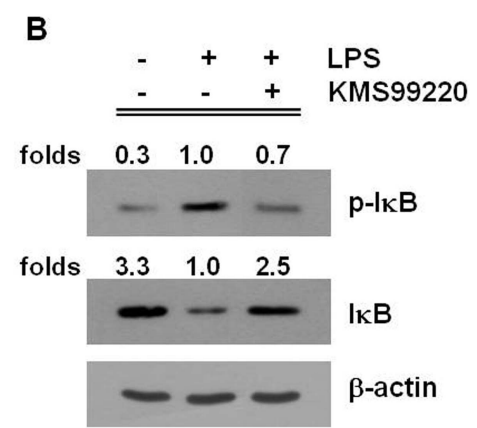

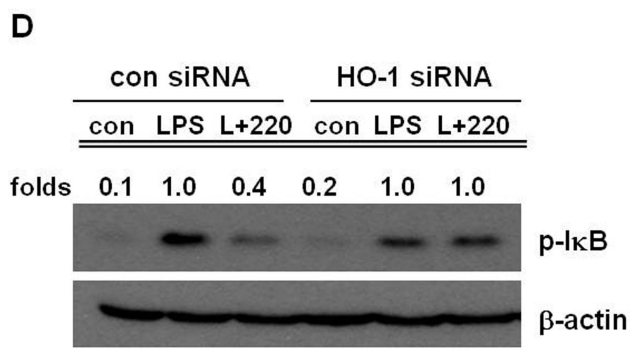

Fig. 3. KMS99220 inhibits activation of $\mathrm{NFKB}$ signaling via $\mathrm{HO}-1$ induction. (A) BV2 cells were treated with $10 \mu \mathrm{M}$ KMS99220 for $1 \mathrm{~h}$, and then treated with $0.2 \mu \mathrm{g} / \mathrm{ml}$ LPS for additional $1 \mathrm{~h}$. Nuclear extracts were isolated and western blot analysis was performed against p65 NFKB. (B) BV2 cells were treated with $10 \mu \mathrm{M}$ KMS99220 for $1 \mathrm{~h}$ and then exposed to $0.2 \mu \mathrm{g} / \mathrm{ml}$ LPS for additional $0.5 \mathrm{~h}$. Cell lysates were analyzed by western blotting with antibodies to $\mathrm{p}$-I $\kappa \mathrm{B}$ or IкB. (C, D) BV2 cells were transfected with siRNA against control or HO-1 for $24 \mathrm{~h}$. (C) Western blot against HO-1 was performed to confirm the knockdown. (D) After treatment with $10 \mu \mathrm{M}$ KMS99220 for $1 \mathrm{~h}$, the HO- 1 knockdown cells were incubated with $0.2 \mu \mathrm{g} /$ $\mathrm{ml}$ LPS for additional $0.5 \mathrm{~h}$ and western blot analysis was performed against p-IкB. (E) After pretreatment with SnPP for $1 \mathrm{~h}, \mathrm{BV} 2$ cells were treated with $10 \mu \mathrm{M}$ KMS99220 for $1 \mathrm{~h}$ followed by $0.2 \mu \mathrm{g} / \mathrm{ml}$ LPS for $0.5 \mathrm{~h}$. Western blot analysis was performed against $\mathrm{p}-\mathrm{I} \kappa \mathrm{B}$. For quantitation, values obtained from densitometry were normalized against respective loading controls (Lamin B or $\beta$-actin) and expressed as fold of respective LPS-treated control (A, B, D, and E) or control siRNAtransfected (C). 


\section{KMS99220 suppresses LPS-induced iNOS expression via HO-1 induction}

Because activated NFкB leads to production of various proinflammatory mediators including NO in microglia, and KMS99220 was found to suppress NFKB activation, we tested whether KMS99220 might also lower NO production. For this, we assessed expression of iNOS, the NO-synthesizing enzyme, and generation of NO in LPS-activated BV2 cells. As shown in Fig. 4A, the mRNA and protein levels of iNOS, which was elevated by LPS, was dosedependently suppressed by the co-treatment with KMS99220. The generation of NO was also suppressed: the LPS-induced NO production was dose-dependently lowered by KMS99220, and 10 $\mu$ M KMS99220 was able to completely block the increase (Fig. 4B).

On the other hand, in the cells whose HO-1 expression was knocked down by transfection with HO-1 siRNA, the inhibitory effect of KMS99220 on the iNOS expression was reversed, and there was no significant difference in the iNOS level between the LPS alone and the LPS+KMS99220 treated cells (Fig. 4C). Pharmacological inhibition of HO-1 with SnPP also dose dependently alleviated the downregulating effect of KMS99220 on iNOS, com- pletely blocking the effect at $10 \mu \mathrm{M}$ SnPP (Fig. 4D). Taken together, the suppression of iNOS expression by KMS99220 appeared to be mediated by HO- 1 .

\section{AMPK mediates the anti-inflammatory effect of KMS99220}

We asked whether AMPK might also play a role in the inhibitory effect of KMS99220 on the NO system. As shown in Fig 5A, pretreatement with the AMPK inhibitor Ara A was able to dose-dependently reverse the inhibitory effect of KMS99220 on the LPSinduced iNOS expression. This correlated well with the amount of NO produced under this condition (Fig. 5B). Compound C, another pharmacological inhibitor of AMPK, showed a similar effect (Fig. 5C). Taken together with the finding that these AMPK inhibitors also suppresses HO-1 induction by KMS99220 (Fig. 2), these results suggested that AMPK likely acts upstream of HO-1 in mediating the anti-inflammatory effect of KMS99220.

\section{KMS99220 can exert early anti-inflammatory effects inde- pendent of $\mathrm{Nrf2}$}

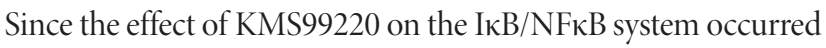

A

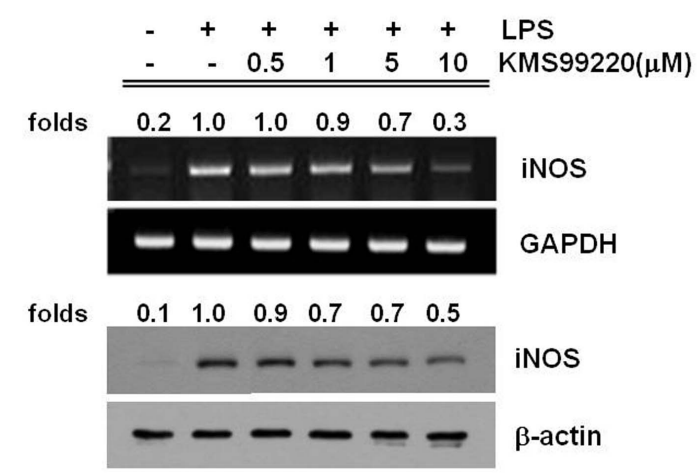

C

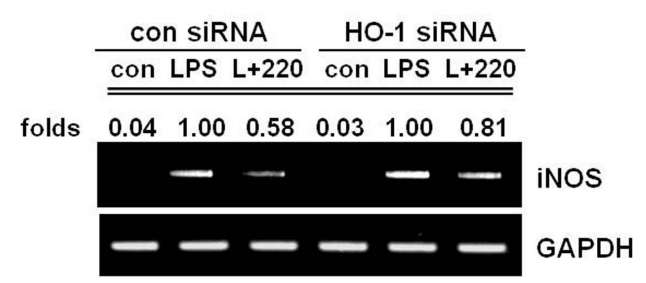

B

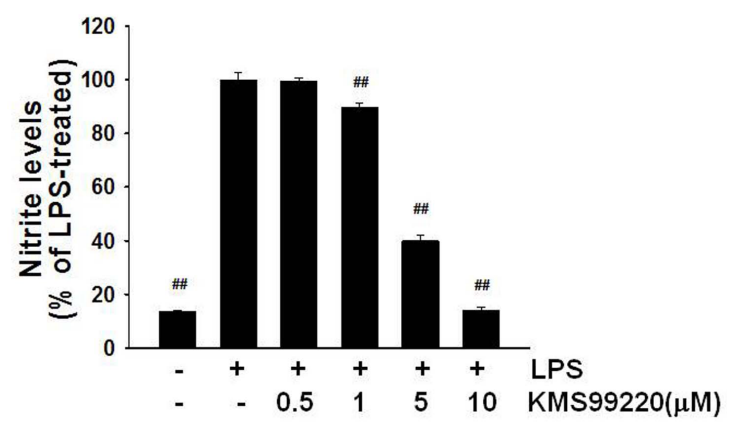

D

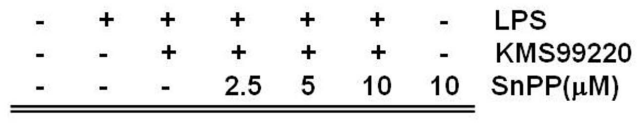

folds

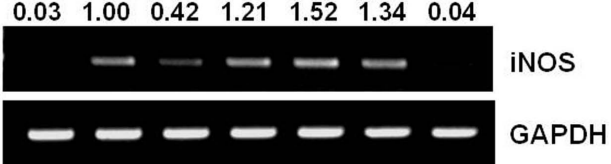

Fig. 4. KMS99220 suppresses iNOS expression via HO- 1 induction. (A, B) BV2 cells were exposed to various concentrations of KMS99220 with $0.2 \mu \mathrm{g} /$ $\mathrm{ml}$ LPS. (A) The cells were harvested after 6 or $24 \mathrm{~h}$, and RT-PCR or western blot analysis was performed against iNOS, respectively. (B) After $24 \mathrm{~h}$, the cell culture medium was subjected to NO determination by the Griess method. The data are expressed as $\%$ of LPS-treated control \pm SEM; ${ }^{* * *} \mathrm{p}<0.01$. $(\mathrm{C})$ After transfection with control or HO-1 siRNA for $24 \mathrm{~h}$ as described for Fig. 3, the cells were treated with $10 \mu \mathrm{M}$ KMS99220 or $0.2 \mu \mathrm{g} / \mathrm{ml} \mathrm{LPS}$ for $6 \mathrm{~h}$. RT-PCR was performed against iNOS using GAPDH as a loading control. (D) BV2 cells were preincubated with SnPP for 1 h, and treated with $10 \mu \mathrm{M}$ KMS99220 and/or $0.2 \mu \mathrm{g} / \mathrm{ml}$ LPS for $6 \mathrm{~h}$. The mRNA level of iNOS was determined by RT-PCR. For quantitative analysis (A, C, and D), values obtained from densitometry were normalized against respective loading controls (GAPDH or $\beta$-actin) and expressed as fold of respective LPS-treated control. 
A

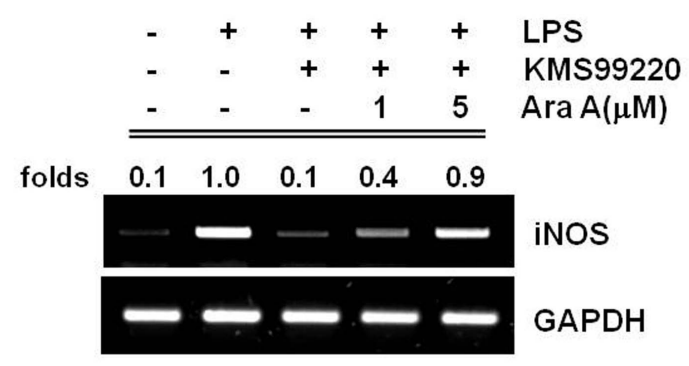

C

\begin{tabular}{llllll}
- & + & + & + & + & LPS \\
- & - & + & + & + & KMS99220 \\
- & - & - & 0.5 & 1 & Compound $C(\mu M)$ \\
\hline
\end{tabular}

folds $\quad \begin{array}{lllll}0.02 & 1.00 & 0.16 & 0.17 & 0.48\end{array}$

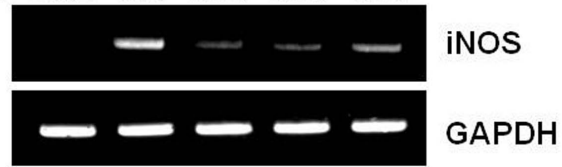

within $1 \mathrm{~h}$, and $\mathrm{HO}-1$ mediated this response, the $\mathrm{HO}-1$ induction would be expected to be increased at an early time point. As shown in Fig. 6A, the HO-1 mRNA level was indeed elevated within 1 $\mathrm{h}$ of the KMS99220 treatment. As the HO- 1 gene is known to be under the control of the transcription factor $\mathrm{Nrf} 2$, it was possible that this early induction occurred via $\mathrm{Nrf} 2$. However, when we examined the expression levels of NQO1, GCLM and GCLC, whose gene expressions are also known to be under the control of Nrf2 signaling, they were not changed in this time frame (Fig. 6A). This suggested that a mechanism independent of Nrf2 signaling might be involved in the KMS99220-induced early expression of HO- 1.

To test this notion, we asked if the KMS99220 effect is still present in cells whose Nrf2 expression has been knocked down. For this, BV-2 cells were transfected with Nrf2 siRNA and the obliteration of Nrf2 mRNA was confirmed (Fig. 6B). When these cells were exposed to KMS99220 for only $1 \mathrm{~h}$ followed by a brief LPS challenge, the increase in the expression of HO-1 was evident, compared to LPS-alone control (Fig. 6C). The degree of HO-1 induction in the Nrf2 knockdown cells was not smaller than that in the control cells. This indicated that the early HO-1 induction observed after KMS99220 exposure indeed did not require Nrf2. Western blot analysis on the same samples against phosphorylated I $\kappa$ B revealed that the inhibition of I B activation by KMS99220 still occurred in the absence of Nrf2 (Fig. 6C). As expected, the subsequent expression of iNOS was still downregulated in the Nrf2
B

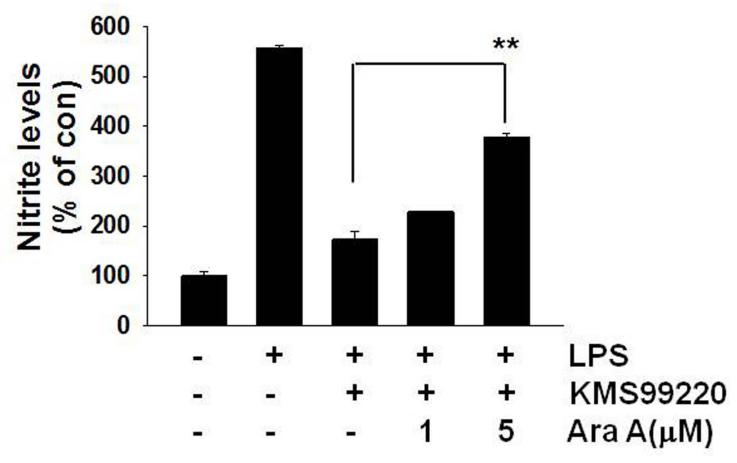

Fig. 5. AMPK mediates the inhibitory effect of KMS99220 on LPS-induced iNOS expression. (A, C) BV2 cells were pretreated with Ara A (A) or Compound C (C) for $1 \mathrm{~h}$, and then treated with $10 \mu \mathrm{M}$ KMS99220 and/or $0.2 \mu \mathrm{g} / \mathrm{ml}$ LPS for $6 \mathrm{~h}$. RT-PCR was performed against iNOS using GAPDH as a loading control. Values obtained from densitometry were normalized against GAPDH and expressed as fold of LPS-treated control. (B) BV2 cells were pretreated with Ara A for $1 \mathrm{~h}$, and then treated with $10 \mu \mathrm{M}$ KMS99220 and/or $0.2 \mu \mathrm{g} / \mathrm{ml}$ LPS for $24 \mathrm{~h}$. Nitrite levels were assessed by the Griess method. The data are expressed as \% of untreated control $\pm \mathrm{SEM} ;{ }^{* *} \mathrm{p}<0.01$.

knockdown cells in a manner not different from the control cells (Fig. 6D). For further confirmation, we performed the same test in BV2 microglia cells whose Nrf2 had been stably knocked down by introducing Nrf2sh [28] (Fig. 6E). Again, KMS99220 effectively induced HO-1 expression and inhibited IкB phosphorylation in the Nrf2 knockdown cells (Fig. 6F). In addition, the downregulation of iNOS expression still occurred in these cells (Fig. 6G). Taken together, these results indicated that the KMS99220-induced early induction of HO-1 and the subsequent anti-inflammatory response occurred independently of the presence of $\mathrm{Nrf} 2$.

\section{DISCUSSION}

With the increasing evidence that neuroinflammation plays a vital role in neurodegeneration, candidate drugs that target neuroinflammation toward therapy for neurodegenerative diseases are being actively sought. In the present study, our novel morpholinecontaining chalcone KMS99220, which was previously shown to possess a neuroprotective property with excellent pharmacological properties [16], also exerts anti-inflammatory effects on microglia, and that this is mediated by AMPK activation followed by HO- 1 induction that occurs independent of the presence of Nrf2.

The present study shows that KMS99220 can suppress activation of NFKB, the inflammation-associated transcription factor, expression of its downstream gene iNOS and production of the 


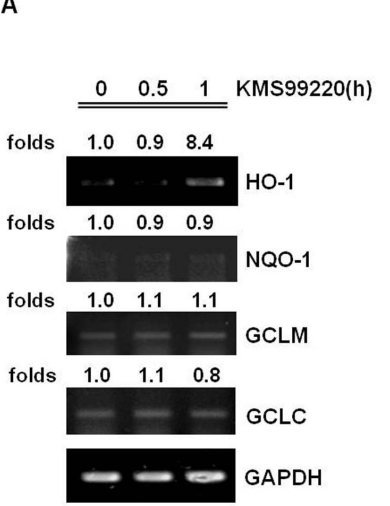

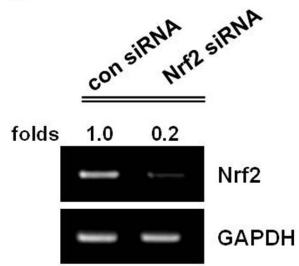

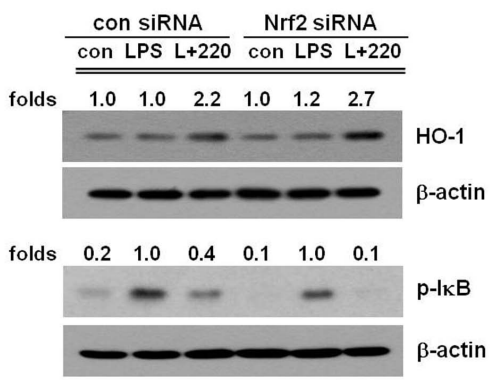

$\mathbf{F}$

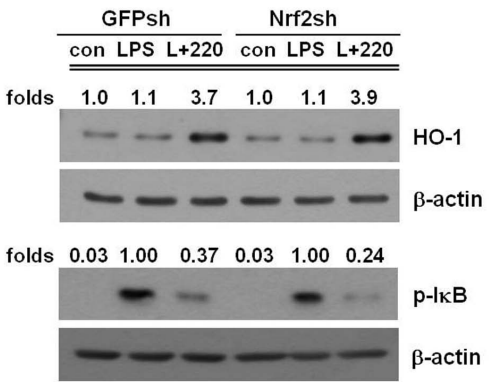

D

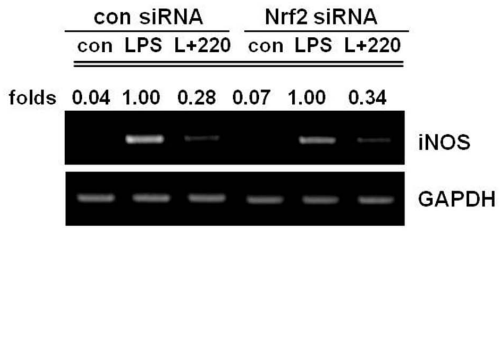

G

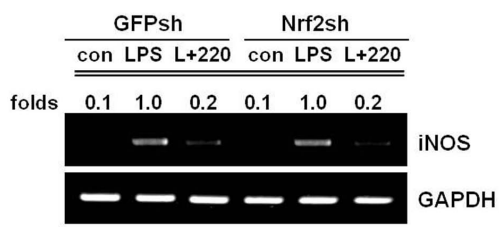

Fig. 6. KMS99220 exerts early anti-inflammatory effects independent of Nrf2. (A) BV2 cells were treated with $10 \mu \mathrm{M}$ KMS99220 for 0.5 or 1 h, and RTPCR was performed against HO-1, NQO1, GCLM and GCLC using GAPDH as a loading control. (B-D) BV2 cells were transfected with siRNA for control or Nrf2 for 24 h. (B) RT-PCR against Nrf2 was performed to confirm the knockdown. (C) The cells were treated with $10 \mu \mathrm{M}$ KMS99220 for 1 $\mathrm{h}$, and then with $0.2 \mu \mathrm{g} / \mathrm{ml}$ LPS for $0.5 \mathrm{~h}$. Western blot analysis was performed against HO- 1 and $\mathrm{p}$-I $\mathrm{KB}$ using $\beta$-actin as a loading control. (D) The Nrf2 knockdown cells were exposed to $10 \mu \mathrm{M}$ KMS99220 and/or $0.2 \mu \mathrm{g} / \mathrm{ml} \mathrm{LPS}$ for $6 \mathrm{~h}$, and RT-PCR was performed against iNOS. (E) RT-PCR against Nrf2 was performed to confirm the knockdown in the GFPsh cells and Nrf2sh cells. (F) GFPsh cells and Nrf2sh cells were treated with $10 \mu \mathrm{M}$ KMS99220 for $1 \mathrm{~h}$, and then with $0.2 \mu \mathrm{g} / \mathrm{ml}$ LPS for $0.5 \mathrm{~h}$. Western blot analysis was performed against HO- 1 and p-IкB. (G) GFPsh cells and Nrf2sh cells were exposed to $10 \mu \mathrm{M}$ KMS99220 and/or $0.2 \mu \mathrm{g} / \mathrm{ml}$ LPS for $6 \mathrm{~h}$, and RT-PCR was performed against iNOS. For quantitation, values obtained from densitometry were normalized against respective GAPDH or $\beta$-actin, and expressed as fold of untreated (A), control siRNA (B), GFPsh (E), or respective LPS-treated control (C, D, F and G).

inflammatory mediator NO. This inhibition of inflammatory signaling by KMS99220 appears to be mediated by HO-1 induction: KMS99220 is able to induce HO-1 gene expression, and the inhibitory effects of KMS99220 on iNOS expression and IкB activation are lost in both HO-1 siRNA-transfected cells and HO-1 inhibitor-treated cells. This is in line with the previous reports on the anti-inflammatory role of HO-1. The exact mechanism of anti-inflammatory activities mediated by $\mathrm{HO}-1$ remains unclear, but the enzymatic metabolites bilirubin and carbon monoxide are thought to be involved. For example, it has been reported that bilirubin inhibits iNOS expression and $\mathrm{NO}$ production in response to endotoxin in rats [32], and that carbon monoxide attenuates NO production and $\mathrm{NF \kappa B}$ activation in LPS-induced endothelial cells [33].

We show in the present study that the early induction of HO-1 expression that occurs within $1 \mathrm{~h}$ of KMS99220 exposure and is associated with the anti-inflammatory response might take place independent of the transcription factor Nrf2. This was demonstrated by the finding that HO- 1 expression and anti-inflammatory responses still occur in the absence of Nrf2. In addition, the expression levels of other Nrf2-dependent genes, such as NQO1, GCLM and GCLC, were not increased in this time frame. We show that the early expression of HO-1 is dependent on AMPK signaling. KMS99220 was able to activate AMPK within 15 min and induce HO- 1 expression, and inhibition of AMPK activity abolished the HO-1 induction. Other transcription factors reported to be involved in HO-1 induction include CREB [34, 35] and FOXO1 $[36,37]$, and they are reported to be activated by AMPK $[38,39]$. Therefore, it is possible to speculate that AMPK, upon activation by KMS99220 and subsequent translocation into the nucleus, acts on any of these transcription factors and leads to the early HO-1 expression.

We have previously shown that KMS99220 is able to bind to Keap1, the inhibitory protein for $\mathrm{Nrf} 2$, and lead to $\mathrm{Nrf} 2$ activation 
[16]. Because $\mathrm{HO}-1$ is a target gene of $\mathrm{Nrf} 2$, it was possible that the KMS99220-induced HO-1 elevation occurred via this signaling. However, we noted a discrepancy in time course, in that the suppression of I $\mathrm{B}$ phosphorylation and $\mathrm{NF} \kappa \mathrm{B}$ nuclear localization by KMS99220 took place faster than the increase in HO-1 resulting from Nrf2 activation. This led us to ask whether there might be another, earlier mechanism for activation of the anti-inflammatory response. The present study shows that AMPK activation and the resulting expression of HO- 1 occurs faster, within the same time window as the NFKB activation. Since KMS99220 also interacts with Keap1, it can be postulated that the KMS99220-induced HO- 1 induction is biphasic, first via AMPK activation and later via Nrf2 signaling (Fig. 7).

KMS99220 appears to act as direct activator of AMPK, because it led to increased enzyme activity of purified AMPK protein. Studies have suggested the utility of AMPK-activating compounds as a therapeutic agent for neuroinflammatory disorders. In microglial cells, pharmacological activation of AMPK using its direct AMPK agonists 5-amino-4-imidazole carboxamide riboside and ENERGI-F704 lowered the LPS-induced production of TNF- $\alpha$, IL- 6 and iNOS and nuclear translocation of NFKB $[2,3]$. In addition, activation of AMPK resulting from exposure to the phytochemicals such as $(+)$ catechin, resveratrol, and lycopene have all been linked

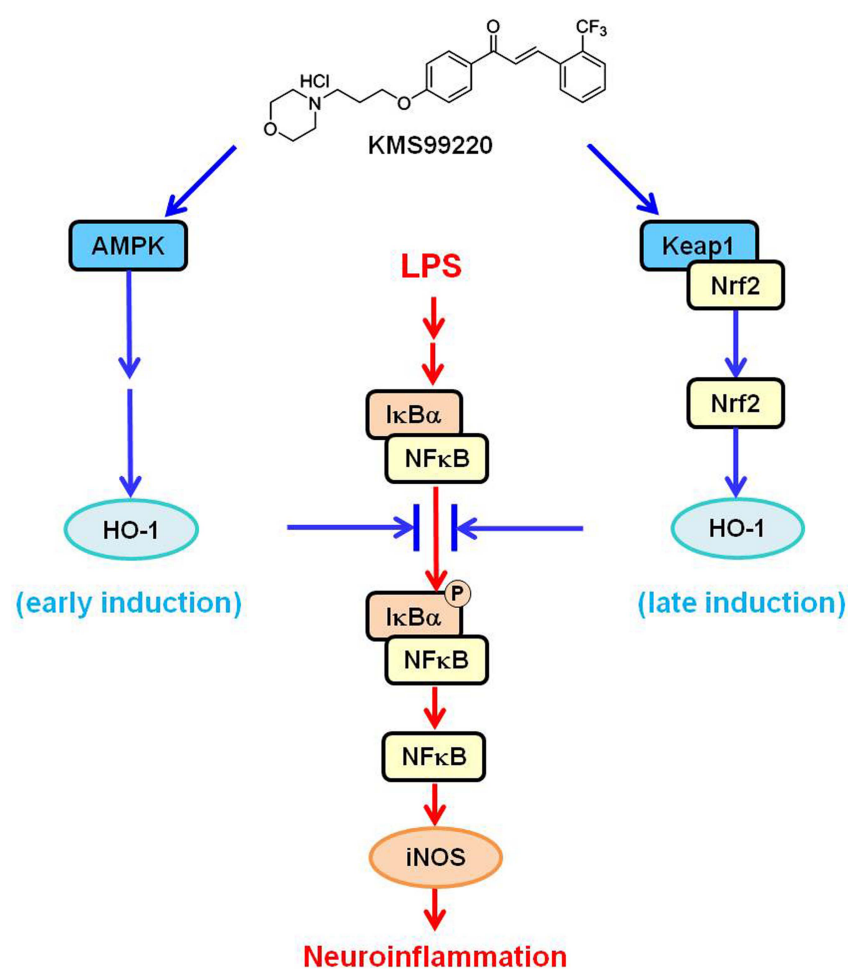

Fig. 7. Proposed mechanism for the anti-inflammatory effects of KMS99220 in microglia. to suppression of microglial activation [13, 40, 41]. In macrophages, overexpression of AMPK results in decreased production of TNF- $\alpha$ and IL- 6 after LPS exposure, whereas knockdown of AMPK expression leads to increased production of these proinflammatory cytokines [4]. In addition, macrophages generated from AMPKal-deficient mice exhibited enhanced inflammatory response [5], and the AMPK signaling downregulates the function of NFKB system $[4,6]$.

In conclusion, our novel chalcone compound KMS99220 activates AMPK signaling, leading to downregulation of inflammatory response in microglia, and this appears to be mediated by HO- 1 that is induced Nrf2-indepdendently downstream of AMPK at an earlier time point. Together with our previous finding that KMS99220 exhibits excellent neuroprotective property and pharmacokinetic profile, the compound might be utilized as a potential candidate for therapy of neuroinflammation- and neurodegeneration-associated disorders.

\section{ACKNOWLEDGEMENTS}

This research was supported by the Korea Health Technology R\&D Project through the Korea Health Industry Development Institute (KHIDI) funded by the Ministry of Health and Welfare (HI15C1799).

\section{REFERENCES}

1. Labzin LI, Heneka MT, Latz E (2018) Innate immunity and neurodegeneration. Annu Rev Med 69:437-449.

2. Chen CC, Lin JT, Cheng YF, Kuo CY, Huang CF, Kao SH, Liang YJ, Cheng CY, Chen HM (2014) Amelioration of LPSinduced inflammation response in microglia by AMPK activation. BioMed Res Int 2014:692061.

3. Giri S, Nath N, Smith B, Viollet B, Singh AK, Singh I (2004) 5-aminoimidazole-4-carboxamide-1-beta-4-ribofuranoside inhibits proinflammatory response in glial cells: a possible role of AMP-activated protein kinase. J Neurosci 24:479-487.

4. Sag D, Carling D, Stout RD, Suttles J (2008) Adenosine 5'-monophosphate-activated protein kinase promotes macrophage polarization to an anti-inflammatory functional phenotype. J Immunol 181:8633-8641.

5. Carroll KC, Viollet B, Suttles J (2013) AMPKal deficiency amplifies proinflammatory myeloid APC activity and CD40 signaling. J Leukoc Biol 94:1113-1121.

6. Yang Z, Kahn BB, Shi H, Xue BZ (2010) Macrophage alphal AMP-activated protein kinase (alpha1AMPK) antagonizes fatty acid-induced inflammation through SIRT1. J Biol Chem 


\section{5:19051-19059.}

7. Poss KD, Tonegawa S (1997) Heme oxygenase 1 is required for mammalian iron reutilization. Proc Natl Acad Sci U S A 94:10919-10924.

8. Kapturczak MH, Wasserfall C, Brusko T, Campbell-Thompson M, Ellis TM, Atkinson MA, Agarwal A (2004) Heme oxygenase- 1 modulates early inflammatory responses: evidence from the heme oxygenase-1-deficient mouse. Am J Pathol 165:1045-1053.

9. Greil J, Verga-Falzacappa MV, Echner NE, Behnisch W, Bandapalli OR, Pechanska P, Immenschuh S, Vijayan V, Balla J, Tsukahara H, Schneider M, Janka G, Claus M, Longerich T, Muckenthaler MU, Kulozik AE (2016) Mutating heme oxygenase- 1 into a peroxidase causes a defect in bilirubin synthesis associated with microcytic anemia and severe hyperinflammation. Haematologica 101:e436-e439.

10. Radhakrishnan N, Yadav SP, Sachdeva A, Pruthi PK, Sawhney S, Piplani T, Wada T, Yachie A (2011) Human heme oxygenase-1 deficiency presenting with hemolysis, nephritis, and asplenia. J Pediatr Hematol Oncol 33:74-78.

11. Weis N, Weigert A, von Knethen A, Brüne B (2009) Heme oxygenase- 1 contributes to an alternative macrophage activation profile induced by apoptotic cell supernatants. Mol Biol Cell 20:1280-1288.

12. Lee EJ, Ko HM, Jeong YH, Park EM, Kim HS (2015) $\beta$-Lapachone suppresses neuroinflammation by modulating the expression of cytokines and matrix metalloproteinases in activated microglia. J Neuroinflammation 12:133.

13. Lin HY, Huang BR, Yeh WL, Lee CH, Huang SS, Lai CH, Lin H, Lu DY (2014) Antineuroinflammatory effects of lycopene via activation of adenosine monophosphate-activated protein kinase- $\alpha 1 /$ heme oxygenase-1 pathways. Neurobiol Aging 35:191-202.

14. Lee DS, Jeong GS (2016) Butein provides neuroprotective and anti-neuroinflammatory effects through Nrf2/AREdependent haem oxygenase 1 expression by activating the PI3K/Akt pathway. Br J Pharmacol 173:2894-2909.

15. Sun GY, Chen Z, Jasmer KJ, Chuang DY, Gu Z, Hannink M, Simonyi A (2015) Quercetin attenuates inflammatory responses in BV-2 microglial cells: role of MAPKs on the Nrf2 pathway and induction of heme oxygenase-1. PLoS One 10:e0141509.

16. Lee JA, Son HJ, Choi JW, Kim J, Han SH, Shin N, Kim JH, Kim SJ, Heo JY, Kim DJ, Park KD, Hwang O (2018) Activation of the Nrf2 signaling pathway and neuroprotection of nigral dopaminergic neurons by a novel synthetic compound KMS99220. Neurochem Int 112:96-107.
17. Mahapatra DK, Bharti SK, Asati V (2017) Chalcone derivatives: anti-inflammatory potential and molecular targets perspectives. Curr Top Med Chem 17:3146-3169.

18. Lee JA, Kim JH, Woo SY, Son HJ, Han SH, Jang BK, Choi JW, Kim DJ, Park KD, Hwang O (2015) A novel compound VSC2 has anti-inflammatory and antioxidant properties in microglia and in Parkinson's disease animal model. Br J Pharmacol 172:1087-1100.

19. Wu J, Li J, Cai Y, Pan Y, Ye F, Zhang Y, Zhao Y, Yang S, Li X, Liang G (2011) Evaluation and discovery of novel synthetic chalcone derivatives as anti-inflammatory agents. J Med Chem 54:8110-8123.

20. Zhang X, Zhu P, Zhang X, Ma Y, Li W, Chen JM, Guo HM, Bucala R, Zhuang J, Li J (2013) Natural antioxidant-isoliquiritigenin ameliorates contractile dysfunction of hypoxic cardiomyocytes via AMPK signaling pathway. Mediators Inflamm 2013:390890.

21. Zhang T, Yamamoto N, Ashida H (2014) Chalcones suppress fatty acid-induced lipid accumulation through a LKB1/ AMPK signaling pathway in HepG2 cells. Food Funct 5:1134-1141.

22. Han JY, Park SH, Yang JH, Kim MG, Cho SS, Yoon G, Cheon SH, Ki SH (2014) Licochalcone suppresses LXRa-induced hepatic lipogenic gene expression through AMPK/Sirtl pathway activation. Toxicol Res 30:19-25.

23. Dang Y, Ling S, Duan J, Ma J, Ni R, Xu JW (2015) Bavachalcone-induced manganese superoxide dismutase expression through the AMP-activated protein kinase pathway in human endothelial cells. Pharmacology 95:105-110.

24. Liu XM, Peyton KJ, Shebib AR, Wang H, Korthuis RJ, Durante W (2011) Activation of AMPK stimulates heme oxygenase-1 gene expression and human endothelial cell survival. Am J Physiol Heart Circ Physiol 300:H84-H93.

25. Zimmermann K, Baldinger J, Mayerhofer B, Atanasov AG, Dirsch VM, Heiss EH (2015) Activated AMPK boosts the $\mathrm{Nrf2/HO-1} \mathrm{signaling} \mathrm{axis--A} \mathrm{role} \mathrm{for} \mathrm{the} \mathrm{unfolded} \mathrm{protein}$ response. Free Radic Biol Med 88:417-426.

26. Gao XY, Wang SN, Yang XH, Lan WJ, Chen ZW, Chen JK, Xie JH, Han YF, Pi RB, Yang XB (2016) Gartanin protects neurons against glutamate-induced cell death in HT22 cells: independence of Nrf-2 but involvement of HO-1 and AMPK. Neurochem Res 41:2267-2277.

27. Blasi E, Barluzzi R, Bocchini V, Mazzolla R, Bistoni F (1990) Immortalization of murine microglial cells by a v-raf/v-myc carrying retrovirus. J Neuroimmunol 27:229-237.

28. Koh K, Cha Y, Kim S, Kim J (2009) tBHQ inhibits LPSinduced microglial activation via Nrf2-mediated suppression 
of p38 phosphorylation. Biochem Biophys Res Commun 380:449-453.

29. Woo SY, Kim JH, Moon MK, Han SH, Yeon SK, Choi JW, Jang BK, Song HJ, Kang YG, Kim JW, Lee J, Kim DJ, Hwang O, Park KD (2014) Discovery of vinyl sulfones as a novel class of neuroprotective agents toward Parkinson's disease therapy. J Med Chem 57:1473-1487.

30. Zaha VG, Young LH (2012) AMP-activated protein kinase regulation and biological actions in the heart. Circ Res 111:800-814.

31. Kawai T, Akira S (2007) Signaling to NF-kappaB by Toll-like receptors. Trends Mol Med 13:460-469.

32. Wang WW, Smith DL, Zucker SD (2004) Bilirubin inhibits iNOS expression and NO production in response to endotoxin in rats. Hepatology 40:424-433.

33. Sun B, Zou X, Chen Y, Zhang P, Shi G (2008) Preconditioning of carbon monoxide releasing molecule-derived CO attenuates LPS-induced activation of HUVEC. Int J Biol Sci 4:270278.

34. Barnett M, Hall S, Dixit M, Arany I (2016) Simvastatin attenuates oleic acid-induced oxidative stress through CREBdependent induction of heme oxygenase- 1 in renal proximal tubule cells. Pediatr Res 79:243-250.

35. Astort F, Repetto EM, Rocha-Viegas L, Mercau ME, Puch SS, Finkielstein CV, Pecci A, Cymeryng CB (2016) Role of CREB on heme oxygenase- 1 induction in adrenal cells: involvement of the PI3K pathway. J Mol Endocrinol 57:113-124.

36. Liu X, Cui Y, Li M, Xu H, Zuo J, Fang F, Chang Y (2013) Cobalt protoporphyrin induces $\mathrm{HO}-1$ expression mediated partially by FOXO1 and reduces mitochondria-derived reactive oxygen species production. PLoS One 8:e80521.

37. Kang J, Jeong MG, Oh S, Jang EJ, Kim HK, Hwang ES (2014) A FoxO1-dependent, but NRF2-independent induction of heme oxygenase-1 during muscle atrophy. FEBS Lett 588:7985.

38. Thomson DM, Herway ST, Fillmore N, Kim H, Brown JD, Barrow JR, Winder WW (2008) AMP-activated protein kinase phosphorylates transcription factors of the CREB family. J Appl Physiol (1985) 104:429-438.

39. Kim TT, Dyck JR (2015) Is AMPK the savior of the failing heart? Trends Endocrinol Metab 26:40-48.

40. Syed Hussein SS, Kamarudin MN, Kadir HA (2015) (+)-Catechin attenuates NF- $\mathrm{kB}$ activation through regulation of Akt, MAPK, and AMPK signaling pathways in LPS-induced BV-2 microglial cells. Am J Chin Med 43:927-952.

41. Han Y, Jiang C, Tang J, Wang C, Wu P, Zhang G, Liu W, Jamangulova N, Wu X, Song X (2014) Resveratrol reduces morphine tolerance by inhibiting microglial activation via AMPK signalling. Eur J Pain 18:1458-1470. 